\title{
Protagonismo dos adolescentes e jovens na prevenção da sua saúde sexual
}

\author{
Protagonism of adolescents and young people in the prevention of their sexual health \\ Protagonismo de adolescentes y jóvenes en la prevención de su salud sexual
}

Recebido: 17/03/2021 | Revisado: 24/03/2021 | Aceito: 29/03/2021 | Publicado: 08/04/2021

\author{
Aíka Barros Barbosa Maia \\ ORCID: https://orcid.org/0000-0001-7682-5824 \\ Fundação Oswaldo Cruz, Brasil \\ E-mail:aikabarbosa@yahoo.com.br \\ Liana Maria Ibiapina do Monte \\ ORCID: https://orcid.org/0000-0002-8339-8477 \\ Fundação Oswaldo Cruz, Brasil \\ E-mail:lianaibiapina@yahoo.com.br \\ Ranieri Flávio Viana de Sousa \\ ORCID: https://orcid.org/0000-0003-3372-0023 \\ Fundação Oswaldo Cruz, Brasil \\ E-mail:ranieriflavio@hotmail.com \\ Alexandro do Vale Silva \\ ORCID: https://orcid.org/0000-0002-5641-0581 \\ Fundação Oswaldo Cruz, Brasil \\ E-mail:alexbioenf@hotmail.com \\ Darwin Renne Florêncio Cardoso \\ ORCID: https://orcid.org/0000-0001-5741-4552 \\ Fundação Oswaldo Cruz, Brasil \\ E-mail:darwin.cardoso@hotmail.com \\ Elaine Ferreira do Nascimento \\ ORCID: https://orcid.org/0000-0002-1632-9148 \\ Fundação Oswaldo Cruz, Brasil \\ E-mail:negraelaine@gmail.com \\ Jacenir Reis dos Santos Mallet \\ ORCID: https://orcid.org/0000-0003-4728-7638 \\ Fundação Oswaldo Cruz, Brasil \\ E-mail:jacemallet@gmail.com
}

\begin{abstract}
Resumo
Objetivou-se descrever evidências cientificas sobre o comportamento e o conhecimento dos adolescentes e jovens sobre a sua sexualidade e as formas de prevenção das infecções sexualmente transmissíveis (IST). Revisão integrativa, com abordagem qualitativa, tendo como questão norteadora: qual o comportamento e o conhecimento dos jovens escolares sobre a sua sexualidade e as formas de prevenção das IST?. A coleta de dados ocorreu no mês de agosto de 2020, a busca foi realizada nas bases de dados da Biblioteca Virtual em Saúde entre elas: Medline, Lilacs e BDENF, utilizando os descritores em Ciências da Saúde (DeCS): Adolescente, Infecção Sexualmente Transmissíveis, Conhecimento e Saúde Sexual. Foram detectados 206 artigos, sendo que 25 atendiam aos critérios de inclusão, após a análise e leitura minuciosa dos escritos. Os comportamentos de risco mais presentes nos estudos da literatura foi a iniciação sexual precoce com o sexo masculino mais vulnerável, acompanhada de práticas sexuais desprotegida, sem adesão neste público ao uso frequente do preservativo, aumentando assim o risco das infecções sexualmente transmissíveis. Observou-se, ainda, um conhecimento limitado dos adolescentes sobre a anatomia e a fisiologia dos órgãos sexuais externos, em especial do feminino.
\end{abstract}

Palavras-chave: Adolescente; Infecção sexualmente transmissíveis; Conhecimento; Saúde sexual.

\begin{abstract}
The objective was to describe scientific evidence about the behavior and knowledge of adolescents and young people about their sexuality and ways of preventing sexually transmitted infections (STIs). Integrative review, with a qualitative approach, with the guiding question: what is the behavior and knowledge of young students about their sexuality and ways of preventing STIs?. Data collection took place in August 2020, the search was carried out in the databases of the Virtual Health Library, among them: Medline, Lilacs and BDENF, using the descriptors in Health Sciences (DeCS): Adolescent, Sexually Transmitted Infection, Knowledge and Sexual Health. 206 articles were detected, 25 of which met the inclusion criteria, after careful analysis and reading of the writings. The risk behaviors most present in the literature studies was early sexual initiation with the most vulnerable male sex, accompanied by unprotected sexual practices, without adherence in this public to the frequent use of condoms, thus increasing the risk of sexually transmitted
\end{abstract}


infections. There was also a limited knowledge among adolescents about the anatomy and physiology of the external sexual organs, especially the female.

Keywords: Adolescent; Sexually transmitted infection; Knowledge; Sexual health.

\section{Resumen}

El objetivo fue describir evidencia científica sobre el comportamiento y conocimiento de adolescentes y jóvenes sobre su sexualidad y formas de prevenir las infecciones de transmisión sexual (ITS). Revisión integradora, con enfoque cualitativo, con la pregunta orientadora: ¿cuál es el comportamiento y conocimiento de los jóvenes estudiantes sobre su sexualidad y formas de prevenir las ITS ?. La recolección de datos tuvo lugar en agosto de 2020, la búsqueda se realizó en las bases de datos de la Biblioteca Virtual en Salud, entre ellas: Medline, Lilacs y BDENF, utilizando los descriptores en Ciencias de la Salud (DeCS): Adolescente, Infección de Transmisión Sexual, Conocimiento y Sexualidad. Salud. Se detectaron 206 artículos, 25 de los cuales cumplieron con los criterios de inclusión, luego de un cuidadoso análisis y lectura de los escritos. Las conductas de riesgo más presentes en los estudios de la literatura fue la iniciación sexual temprana con el sexo masculino más vulnerable, acompañada de prácticas sexuales desprotegidas, sin apego en este público al uso frecuente del condón, aumentando así el riesgo de infecciones de transmisión sexual. También existía un conocimiento limitado entre los adolescentes sobre la anatomía y fisiología de los órganos sexuales externos, especialmente la mujer.

Palabras clave: Adolescente; Infección transmitida sexualmente; Conocimiento; Salud sexual.

\section{Introdução}

A Organização Mundial de Saúde (OMS) define adolescência como sendo o período da vida que começa aos 10 anos e termina aos 19 anos completos. Para a OMS, a adolescência é dividida em três fases: Pré-adolescência - dos 10 aos 14 anos, Adolescência - dos 15 aos 19 anos completos, Juventude - dos 15 aos 24 anos. No Brasil, o Estatuto da Criança e do Adolescente (ECA) considera a adolescência, a faixa etária dos 12 até os 18 anos de idade completos (Farias et al., 2009).

Nesta fase da vida, ocorre aceleração e desaceleração do crescimento físico, mudança da composição corporal, eclosão hormonal, envolvendo hormônios sexuais e evolução da maturidade sexual, acompanhada pelo desenvolvimento de caracteres sexuais secundários masculinos e femininos. Paralelamente às mudanças corporais, ocorrem as psicoemocionais, como a busca da identidade, a tendência grupal, o desenvolvimento do pensamento conceitual, a vivência singular e a evolução da sexualidade (Soares, Amaral, Silva \& Silva, 2008).

As transformações dessa fase da vida fazem com que o adolescente viva intensamente sua sexualidade, manifestandoa muitas vezes através de práticas sexuais desprotegidas, podendo se tornar um problema devido à falta de informação, de comunicação entre os familiares, tabus ou mesmo pelo fato de ter medo de assumi-la. A evolução de suas sensações, comportamentos e decisões sexuais será influenciada pelas interações que desenvolve com outros jovens do seu vínculo familiar e social (Araújo, 2001; Castro, Abramovay \& Silva, 2014).

A adolescência corresponde a uma etapa da vida que cresce a autonomia e a independência em relação à família e a experimentação de novos comportamentos e vivências que podem representar importantes fatores de risco para a saúde, como o sexo desprotegido, o uso de drogas lícitas e/ou ilícitas, entre elas, o consumo de álcool e tabaco. Fatores que predispõe o surgimento de infecções por doenças sexualmente transmissíveis, gravidez indesejada, acidentes e violências (Sociedade Brasileira de Pediatria, 2018).

A Organização Mundial da Saúde revela que grande parte dos adolescentes inicia sua vida sexual cada vez mais cedo, a maioria entre os 12 aos 17 anos.3 Muitas vezes o fazem sem a menor informação sobre práticas de sexo mais seguro adotando práticas e/ou comportamentos sexuais que os deixam sob maior vulnerabilidade para infecção com as Infecções sexualmente transmissíveis (IST), o Vírus da imunodeficência humana (HIV) e a Síndrome da imunodeficiência adquirida (AIDS) (Moura, Torres, Cadete \& Freitas Cunha, 2018).

A taxa de crescimento de pessoas com idades de 15 a 19 anos com HIV passou de aproximadamente 800.000 em 2005 para 940.000 em 2015 em 25 países pesquisados, confirmando a alta incidência nessa população.10 No Brasil, em 2015, 
registrou-se recorde de pessoas em tratamento de HIV/Aids. Só neste ano, 81 milhões iniciaram o tratamento com antirretroviral.9 Mesmo assim, pesquisas apontam que mesmo com a presença massificada de campanhas de mídia relacionadas à transmissão de infecções através do sexo como HIV/Aids, não são eficazes suficientemente para cessar todas as dúvidas sobre prevenção e transmissão (Secretaria de Vigilância em Saúde, 2016; Joint United Nations Program on HIV/AIDS, 2016).

Dados do Ministério da Saúde apontam que, entre os homens, nos últimos dez anos, observou-se um aumento da taxa de detecção de aids na faixa etária de 15 a 19 anos, 20 a 24 anos, 25 e 29 anos e 60 anos e mais. Destaca-se o aumento em jovens de 15 a 19 anos e de 20 a 24 anos: do ano de 2006 para o de 2016, a taxa quase triplicou entre o primeiro grupo e, entre os de 20 a 24 anos, a taxa mais que duplicou (Boletim Epidemiológico de AIDS, HIV e DST, 2019).

Portanto, ressalta-se que antes da iniciação da vida sexual, o indivíduo no início da adolescência já tenha a conscientização, responsabilidade e cuidados com a sua saúde sexual e reprodutiva, voltado para a prevenção as IST. Atentar para a sexualidade dos adolescentes é uma necessidade que pode contribuir para reduzir problemas no que diz respeito à sua vida pessoal e social., porém para isso se faz necessário conhecer o perfil destes jovens sobre a sua saúde sexual e as formas de proteção e cuidado, por isso ressalta-se a importância desta pesquisa.

Com isso o objetivo deste estudo foi analisar a partir da literatura, as evidencias cientificas sobre o comportamento e o conhecimento dos adolescentes e jovens sobre a sua sexualidade e as formas de prevenção das IST, para assim desenvolver uma reflexão crítica reflexiva a cerca desta temática.

\section{Metodologia}

O estudo corresponde a uma revisão integrativa de abordagem qualitativa, que para o desenvolvimento do estudo, foram realizadas seis etapas: 1. Identificação do tema e seleção da questão de pesquisa; 2. Estabelecimento de critérios de inclusão e exclusão; 3. Identificação dos estudos pré-selecionados e selecionados; 4. Categorização dos estudos selecionados; 5. Análise e interpretação dos resultados e 6. Apresentação da revisão/síntese do conhecimento (Mendes, Silveira \& Galvão, 2008)

A questão norteadora do estudo foi: qual o comportamento e conhecimento dos adolescentes e jovens sobre a sua sexualidade e as formas de prevenção das IST?

Na realização da pesquisa foram seguidos critérios de inclusão elencados: artigos disponíveis online e na íntegra, no período de 2010 a 2020, escritos em português, dentro da temática do estudo. Foram excluídos artigos duplicados, fora do período estabelecido e com objeto e temática de estudo incompatíveis à proposta deste estudo.

A coleta de dados ocorreu no mês de agosto de 2020, a busca foi realizada nas bases de dados da Biblioteca Virtual em Saúde entre elas: Medline, Lilacs e BDENF, garantindo rigor ao processo de seleção dos artigos nas bases de dados com descritores padronizados e disponíveis nos Descritores em Ciências da Saúde (DeCS): "Adolescente" [and] "Infecção Sexualmente Transmissíveis" [and] "Conhecimento" [and] "Saúde Sexual".

Obteve-se como resultados e análise nas bases de dados 206 artigos, porém apenas 90 atendiam aos critérios de inclusão, compatíveis a temática de estudo filtrou-se 42 artigos, após a leitura completa a amostra final foi constituída por 25 artigos. Em seguida procedeu-se a análise dos dados, delimitando-se as variáveis para análise e discussão dos dados: ano de publicação/autores, títulos, objetivo do estudo, metodologia aplicada, e os resultados encontrados.

Após a análise, foi realizada a discussão dos resultados com a classificação de categorias por similaridade semântica, e as temáticas foram agrupadas conforme semelhança de conteúdo, sendo formadas as categorias de análise temática que foi caracterizada e analisada. 


\section{Resultados e Discussão}

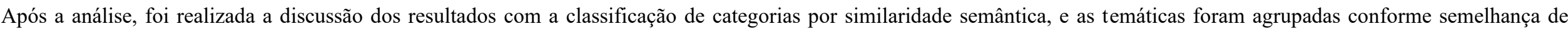
conteúdo, sendo formadas as categorias de análise temática que foi caracterizada e analisada.

Tabela 1. Descrição dos artigos de acordo com os principais domínios sobre o conhecimento dos jovens escolares sobre a sua sexualidade e as formas de prevenção e saúde.

\begin{tabular}{|c|c|c|c|}
\hline Titulo Do Artigo & Objetivo Do Estudo & Delineamento & Resultado \\
\hline $\begin{array}{l}\text { 1. Alves e Aguiar, 2020, saúde } \\
\text { sexual e infecções sexualmente } \\
\text { transmissíveis na adolescência: } \\
\text { uma revisão integrativa }\end{array}$ & $\begin{array}{l}\text { Avaliar o conhecimento e o comportamento } \\
\text { sexual dos adolescentes acerca das infecções sexualmente } \\
\text { transmissíveis. }\end{array}$ & Revisão integrativa da literatura & $\begin{array}{l}\text { Demostraram déficit no conhecimento dos adolescentes acerca } \\
\text { das infecções sexualmente transmissíveis, bem como não } \\
\text { utilização do preservativo de modo rotineiro devido } \\
\text { acreditarem que este inibe o prazer sexual }\end{array}$ \\
\hline $\begin{array}{l}\text { 2. Costa et al., } 2019 \text {, } \\
\text { Determinantes sociais da saúde e } \\
\text { vulnerabilidades às infecções } \\
\text { sexualmente transmissíveis em } \\
\text { adolescentes. }\end{array}$ & $\begin{array}{l}\text { Verificar a associação entre determinantes sociais de saúde e } \\
\text { a vulnerabilidade de adolescentes às Infecções Sexualmente } \\
\text { Transmissíveis (IST). }\end{array}$ & $\begin{array}{l}\text { Estudo transversal, realizado com } \\
287 \text { escolares de } 11 \text { a } 17 \text { anos, na } \\
\text { periferia de Fortaleza, Ceará, }\end{array}$ & $\begin{array}{l}\text { Adolescentes foram considerados mais vulneráveis, com } \\
\text { escore } \geq 4 \text {. O determinante social intermediário } \\
\text { " habitação (casa própria)" obteve associação significativa com } \\
\text { a vulnerabilidade a IST }\end{array}$ \\
\hline $\begin{array}{l}\text { 3. Brasil, Cardoso e Silva, } 2019 \text {, } \\
\text { Conhecimento de escolares sobre } \\
\text { infecções sexualmente } \\
\text { transmissíveis e métodos } \\
\text { contraceptivos }\end{array}$ & $\begin{array}{l}\text { Avaliar o nível de conhecimento de escolares } \\
\text { sobre Infecções Sexualmente Transmissíveis e métodos } \\
\text { contraceptivos. }\end{array}$ & $\begin{array}{l}\text { Trata-se de um estudo qualitativo, } \\
\text { tipo pesquisa-ação, com } 153 \text { escolares } \\
\text { na faixa etária dos } 11 \text { aos } 16 \text { anos, }\end{array}$ & $\begin{array}{l}\text { Revela-se que } 94,1 \% \text { dos discentes disseram saber, pelo } \\
\text { menos, uma maneira de prevenir-se de uma gravidez, sendo } \\
\text { a camisinha masculina conhecida por } 86,9 \% \text { dos entrevistados } \\
\text { e a "pílula do dia seguinte", por } 80,4 \% \text {. Pontua-se, sobre o que } \\
\text { são as infecções sexualmente transmissíveis, que } 15,7 \% \text { não } \\
\text { souberam responder; } 22,9 \% \text { afirmaram não conhecer nenhum } \\
\text { meio de prevenção; } 61,4 \% \text { não souberam relatar nenhum } \\
\text { possível sintoma; } 24,2 \% \text { declararam desconhecer os } \\
\text { agravamentos se não tratados e } 41,9 \% \text { dos entrevistados } \\
\text { disseram achar possível estar contaminados com alguma, sem } \\
\text { ter o conhecimento desse fato }\end{array}$ \\
\hline $\begin{array}{l}\text { 4. Lima, 2019, Práticas de } \\
\text { prevenção de infecções } \\
\text { sexualmente transmissíveis de } \\
\text { universitários do sexo masculino: } \\
\text { estudo comparativo }\end{array}$ & $\begin{array}{l}\text { Analisar comparativamente as práticas } \\
\text { de prevenção das infecções sexualmente transmissíveis (IST) } \\
\text { de jovens universitários do sexo masculino de } \\
\text { duas instituições. }\end{array}$ & $\begin{array}{l}\text { Estudo quantitativo, descritivo, } \\
\text { transversal, desenvolvido em } \\
\text { duas instituições de ensino } \\
\text { superior (IES1 e IES2), no Rio de } \\
\text { Janeiro, com amostra do tipo } \\
\text { intencional e estratificada, de } \\
768 \text { jovens universitários } \\
\text { do sexo masculino }\end{array}$ & $\begin{array}{l}\text { Em relação ao conhecimento sobre as IST, embora } \\
\text { os jovens tenham informado que não conhecem o suficiente, } \\
\text { reconhecem algum método para a prevenção das infecções. }\end{array}$ \\
\hline
\end{tabular}




\begin{tabular}{|c|c|c|c|}
\hline $\begin{array}{l}\text { 5. Felisbino-Mendes, Paula, } \\
\text { Machado, Oliveira-Campos e } \\
\text { Malta, 2018, Análise dos } \\
\text { indicadores de saúde sexual e } \\
\text { reprodutiva de adolescentes } \\
\text { brasileiros, 2009, 2012 e } 2015\end{array}$ & $\begin{array}{l}\text { Analisar saúde sexual e } \\
\text { reprodutiva indicadores de adolescentes com base nos dados } \\
\text { da Pesquisa Nacional Escola baseados Health } \\
\text { Survey (PeNSE) em 2015, }\end{array}$ & $\begin{array}{l}\text { Estudo transversal que analisou dados } \\
\text { de alunos do } 9^{\circ} \text { ano da PeNSE } 2015 \text {, } \\
2012 \text { e } 2009 \text {. }\end{array}$ & $\begin{array}{l}\text { A prevalência de iniciação sexual relatada } \\
\text { por adolescentes diminuiu de } 30,5 \% \text {, em } 2009 \text {, para } 27,5 \% \text {, em } \\
2015 \text {, assim como o uso de preservativo na última relação } \\
\text { sexual, de } 75,9 \text { para } 66,2 \% \text {, } \\
\text { respectivamente. Em relação ao aconselhamento, houve } \\
\text { redução na prevenção da gravidez nas escolas públicas , de } \\
81,1 \text { para } 79,3 \% \text {, em relação } \\
\text { ao preservativo gratuito nas escolas privadas, de } 65,4 \text { para } \\
57,3 \% \text {. Cerca de } 30 \% \text { relataram usar preservativo e } \\
\text { outro método anticoncepcional e } 19,5 \% \text { não usam } \\
\text { nenhum método }\end{array}$ \\
\hline $\begin{array}{l}\text { 6. Sousa et al., } 2018 \text {, } \\
\text { comportamento sexual e fatores } \\
\text { associados em adolescentes rurais. }\end{array}$ & $\begin{array}{l}\text { Descrever o comportamento sexual e identificar fatores } \\
\text { associados em adolescentes de comunidades rurais da } \\
\text { Bahia, Brasil }\end{array}$ & $\begin{array}{l}\text { Trata-se de um estudo transversal, } \\
\text { de base populacional e domiciliar, } \\
\text { realizado em } 2015 \\
\text { com adolescentes de } 10 \text { a } 19 \text { anos. }\end{array}$ & $\begin{array}{l}\text { Foram entrevistados } 390 \text { adolescentes, sendo } 42,8 \% \\
\text { quilombolas, } 51,3 \% \text { do sexo feminino e a mediana de idade de } \\
14,8 \text { anos. Destes adolescentes, } 26,4 \% \text { relataram relação } \\
\text { sexual ( } 28,1 \% \text { quilombolas e } 25,1 \% \text { não quilombolas), e a } \\
\text { mediana de idade da primeira relação foi de } 15 \text { anos; } 77,7 \% \\
\text { delas mencionaram o uso de preservativo na última relação } \\
\text { sexual e mais da metade recebeu orientações } \\
\text { sobre gravidez, AIDS ou outras doenças sexualmente } \\
\text { transmissíveis e nenhuma orientação sobre como } \\
\text { conseguir preservativos gratuitamente }\end{array}$ \\
\hline $\begin{array}{l}\text { 7. Baldoino, Silva, Ribeiro e } \\
\text { Ribeiro, 2018, Educação em } \\
\text { saúde para adolescentes no } \\
\text { contexto escolar: um relato de } \\
\text { experiência }\end{array}$ & $\begin{array}{l}\text { Relatar a experiência de discentes do curso de Bacharelado } \\
\text { em Enfermagem em práticas de educação em } \\
\text { saúde aos adolescentes no contexto escolar. }\end{array}$ & $\begin{array}{l}\text { Estudo descritivo do tipo relato de } \\
\text { experiência }\end{array}$ & $\begin{array}{l}\text { a experiência constituiu oportunidade de realização } \\
\text { da educação em saúde a adolescentes, favorecendo habilidades } \\
\text { e disseminação de conhecimentos }\end{array}$ \\
\hline $\begin{array}{l}\text { 8. Oliveira, Abud, Inagaki, Alves } \\
\text { e Matos, 2018, Vulnerabilidade de } \\
\text { adolescentes às doenças } \\
\text { sexualmente transmissíveis na } \\
\text { atenção primária }\end{array}$ & $\begin{array}{l}\text { Identificar as situações de vulnerabilidade em que } \\
\text { os adolescentes se encontram em relação às Doenças } \\
\text { Sexualmente Transmissíveis na Atenção Primária à Saúde }\end{array}$ & $\begin{array}{l}\text { Estudo bibliográfico, descritivo, } \\
\text { tipo revisão integrativa }\end{array}$ & $\begin{array}{l}\text { Os resultados identificaram as seguintes temáticas condições } \\
\text { socioeconômicas; início precoce da atividade sexual; falta do } \\
\text { uso do preservativo; diferença de gêneros; e dificuldade } \\
\text { de comunicação e acesso aos serviços de Atenção Primária à } \\
\text { Saúde. }\end{array}$ \\
\hline $\begin{array}{l}\text { 9. Silva, Cortes, Espirito Santo e } \\
\text { Cordeiro, 2017, Pesquisa-ação: } \\
\text { promovendo educação em saúde } \\
\text { com adolescentes sobre infecção } \\
\text { sexualmente transmissível }\end{array}$ & $\begin{array}{l}\text { Identificar as dúvidas dos alunos de uma escola pública } \\
\text { federal sobre Infecção Sexualmente Transmissível e propor } \\
\text { uma abordagem ou metodologia educacional mais } \\
\text { apropriada para os alunos. }\end{array}$ & $\begin{array}{l}\text { Estudo qualitativo, descritivo e } \\
\text { exploratório, do tipo pesquisa-ação }\end{array}$ & $\begin{array}{l}127 \text { alunos convidados, compareceram } 81 \text { alunos ( } 64 \%) ; 69 \% \\
\text { deles disseram que sabiam o que é IST e } 41 \% \text { não sabiam } \\
\text { definir. Ao serem questionados sobre com quem gostariam de } \\
\text { aprender sobre IST, escolheram os profissionais de educação e } \\
\text { os de saúde }\end{array}$ \\
\hline
\end{tabular}




\begin{tabular}{|c|c|c|c|}
\hline $\begin{array}{l}\text { 10. Torquato et al., } 2017 \text {, } \\
\text { Adolescentes escolares acerca das } \\
\text { DST/AIDS: quando o } \\
\text { conhecimento não acompanha as } \\
\text { práticas seguras }\end{array}$ & $\begin{array}{l}\text { Avaliar os saberes e as práticas dos adolescentes escolares } \\
\text { em relação às DSTs/AIDS. }\end{array}$ & $\begin{array}{l}\text { Estudo transversal de abordagem } \\
\text { quantitativa em que se aplicou } \\
\text { um questionário do tipo } \\
\text { inquérito Conhecimento, Atitude e } \\
\text { Prática, do Ministério da Saúde, }\end{array}$ & $\begin{array}{l}\text { a maioria dos entrevistados não possuía } \\
\text { o conhecimento adequado sobre as DSTs/AIDS. As práticas } \\
\text { são preocupantes, como a iniciação sexual precoce somada às } \\
\text { práticas sexuais dinâmicas. }\end{array}$ \\
\hline $\begin{array}{l}\text { 11. Cordeiro, Santos, Sales, } \\
\text { Morais e Dutra, } 2017 \text {, Fatores de } \\
\text { risco e de proteção entre } \\
\text { adolescentes em relação às } \\
\text { DST/HIV/AIDS }\end{array}$ & $\begin{array}{l}\text { Investigar entre os adolescentes os fatores de risco e } \\
\text { de proteção em relação às DST/HIV/Aids. }\end{array}$ & $\begin{array}{l}\text { Estudo descritivo, de abordagem } \\
\text { qualitativa, }\end{array}$ & $\begin{array}{l}\text { Evidenciou-se que os adolescentes reconhecem o uso do } \\
\text { preservativo, porém, muitos não o fazem, adotando } \\
\text { comportamentos de risco. O diálogo foi assimilado } \\
\text { positivamente, sendo um fator de proteção. }\end{array}$ \\
\hline $\begin{array}{l}\text { 12. Bordignon, Liberali e } \\
\text { Bordignon, 2017, Causas da não } \\
\text { utilização de preservativos nas } \\
\text { práticas sexuais de adolescentes: } \\
\text { revisão integrativa }\end{array}$ & $\begin{array}{l}\text { Identificar as principais causas para a não utilização } \\
\text { dos métodos de barreira nas práticas sexuais } \\
\text { dos adolescentes }\end{array}$ & Revisão integrativa & $\begin{array}{l}\text { A principal causa encontrada é a dificuldade de negociação da } \\
\text { utilização do preservativo com o parceiro. }\end{array}$ \\
\hline $\begin{array}{l}\text { 13. Almeida et al., } 2017 \text {, } \\
\text { Conhecimento de adolescentes } \\
\text { sobre infecções sexualmente } \\
\text { transmissíveis e gravidez }\end{array}$ & $\begin{array}{l}\text { Investigar o conhecimento de adolescentes em relação } \\
\text { às doenças sexualmente } \\
\text { transmissíveis (IST), AIDS e gravidez, e compreender } \\
\text { o papel da escola na educação sexual. }\end{array}$ & Estudo qualitativo descritivo, & $\begin{array}{l}\text { Os adolescentes reconhecem a importância da educação } \\
\text { sexual; portanto, é importante } \\
\text { implementar estratégiaspromover e proteger } \\
\text { a saúde no ambiente escolar para estimular e fortalecer o } \\
\text { autocuidado em saúde }\end{array}$ \\
\hline $\begin{array}{l}\text { 14Silva et al., } 2016 \text {, } \\
\text { Conhecimento de estudantes } \\
\text { adolescentes sobre } \\
\text { transmissão,prevenção e } \\
\text { comportamentos de risco em } \\
\text { relação as DST/HIV/AIDS }\end{array}$ & $\begin{array}{l}\text { Avaliar o conhecimento de adolescentes, estudantes de } \\
\text { uma escola pública na cidade de Natal/RN, } \\
\text { sobre transmissão, prevenção e comportamentos de risco em } \\
\text { relação às DST/HIV/AIDS. }\end{array}$ & $\begin{array}{l}\text { Estudo descritivo-exploratório, com } \\
\text { abordagem quantitativa, }\end{array}$ & $\begin{array}{l}\text { O estudo apontou índices significativos de desconhecimento } \\
\text { em relação à transmissão, prevenção e tratamento da AIDS e } \\
\text { elucidou alguns comportamentos de risco que tornam } \\
\text { a população jovem vulnerável às DST/AIDS. }\end{array}$ \\
\hline $\begin{array}{l}\text { 15. Silva, Jacob e Hirdes, } 2015 \text {, } \\
\text { Conhecimento de adolescentes do } \\
\text { ensino médio sobre DST/AIDS no } \\
\text { sul do Brasil }\end{array}$ & $\begin{array}{l}\text { Investigar } \\
\text { o conhecimento sobre DST/AIDS em adolescentes de Ensino } \\
\text { Médio de Escolas Públicas Estaduais de Charqueadas/RS. }\end{array}$ & Estudo descritivo e qualitativo & $\begin{array}{l}\text { Os resultados mostram que os adolescentes têm } \\
\text { o conhecimento sobre o que são as DST/AIDS, suas formas } \\
\text { de transmissão na relação sexual, preservativos são usados } \\
\text { como proteção e que algumas DST não têm cura. Porém, eles } \\
\text { têm dúvidas quanto às formas de transmissão sem o contato } \\
\text { sexual, à vulnerabilidade pelo não uso do preservativo, } \\
\text { ao consumo de bebidas alcoólicas e de drogas } \\
\text { ilícitas. Pais e amigos são fontes } \\
\text { de conhecimento sobre DST/AIDS fora da escola }\end{array}$ \\
\hline $\begin{array}{l}\text { 16. Sasaki et al., } 2014 \text {, } \\
\text { Comportamento sexual de } \\
\text { adolescentes }\end{array}$ & $\begin{array}{l}\text { Investigar as características do comportamento sexual de } \\
\text { adolescentes escolares e verificar se há diferenças em } \\
\text { relação ao sexo dos estudantes e ao tipo de escola. }\end{array}$ & $\begin{array}{l}\text { Estudo transversal utilizando dados da } \\
\text { Pesquisa Nacional de Saúde do Escolar } \\
\text { (PeNSE) 2009, }\end{array}$ & $\begin{array}{l}\text { A maioria teve a primeira relação com } 13 \text { anos ou menos, com } \\
\text { até } 3 \text { parceiros, utilizou algum método contraceptivo na última } \\
\text { relação e recebeu orientação sobre prevenção na escola. } \\
\text { A idade da primeira relação foi mais precoce e o número de }\end{array}$ \\
\hline
\end{tabular}




\begin{tabular}{|c|c|c|c|}
\hline $\begin{array}{l}\text { escolares da cidade de Goiânia, } \\
\text { Goiás }\end{array}$ & & & $\begin{array}{l}\text { parceiros foi mais elevado entre os meninos. O relato } \\
\text { de orientações recebidas sobre prevenção de gravidez foi mais } \\
\text { frequente entre meninas e nas instituições privadas. Nestas, foi } \\
\text { também mais elevado o relato de orientações sobre DST/AIDS }\end{array}$ \\
\hline $\begin{array}{l}\text { 17. Oliveira-Campos et al., 2014, } \\
\text { Comportamento sexual em } \\
\text { adolescentes brasileiros, Pesquisa } \\
\text { Nacional de Saúde do Escolar } \\
\text { (PeNSE 2012) }\end{array}$ & $\begin{array}{l}\text { Este estudo descreve o comportamento sexual entre } \\
\text { estudantes que participaram da Pesquisa Nacional sobre a } \\
\text { Saúde do Escolar (PeNSE) } 2012\end{array}$ & $\begin{array}{l}\text { Estudo transversal utilizando dados da } \\
\text { Pesquisa Nacional de Saúde do Escolar } \\
\text { (PeNSE) } 2012\end{array}$ & $\begin{array}{l}\text { Mais de um quarto dos adolescentes já tiveram relação sexual } \\
\text { na vida, sendo mais frequente entre os meninos. Cerca de } 25 \% \\
\text { não fizeram uso de preservativo na última relação sexual. } \\
\text { Baixa escolaridade materna e trabalhar aumentaram a chance } \\
\text { de comportamento sexual de risco. Tanto a chance de sexo } \\
\text { protegido quanto de desprotegido aumentou com o número de } \\
\text { substâncias psicoativas utilizadas. Entre os que não recebem } \\
\text { orientação sobre prevenção de gravidez na escola, a chance ter } \\
\text { relação sexual aumentou, sendo a magnitude maior para sexo } \\
\text { desprotegido }(\mathrm{OR}=1,87) \text {. }\end{array}$ \\
\hline $\begin{array}{l}\text { 18. Malta et al., 2011, Saúde } \\
\text { sexual dos adolescentes segundo a } \\
\text { Pesquisa Nacional de Saúde dos } \\
\text { Escolares. }\end{array}$ & $\begin{array}{l}\text { Descrever as situações relacionadas à saúde sexual dos } \\
\text { adolescentes, segundo a Pesquisa Nacional de Saúde do } \\
\text { Escolar (PeNSE). }\end{array}$ & Trata-se de um estudo transversal & $\begin{array}{l}\text { Aproximadamente um terço dos adolescentes já haviam tido } \\
\text { relação sexual alguma vez na vida, sendo mais meninos do que } \\
\text { meninas e mais aqueles que estudam em escola pública. A } \\
\text { idade da relação sexual foi precoce, e a maioria relatou ter tido } \\
\text { um único parceiro na vida. O uso do preservativo e de método } \\
\text { contraceptivo foi elevado. }\end{array}$ \\
\hline $\begin{array}{l}19 \text { Souza, } 2011 \text {, Adolescentes em } \\
\text { cena: uma proposta educativa no } \\
\text { campo da saúde sexual e } \\
\text { reprodutiva }\end{array}$ & $\begin{array}{l}\text { Descrever a experiência sobre a elaboração de material } \\
\text { educativo, no formato de performance teatral criada e } \\
\text { encenada por adolescentes, como estratégia para a obtenção } \\
\text { de uma atitude reflexiva e autônoma desses sujeitos, no } \\
\text { campo afetivo-sexual e reprodutivo. }\end{array}$ & Relato de experiência & $\begin{array}{l}\text { Observou-se, ainda, um conhecimento limitado dos } \\
\text { adolescentes sobre a anatomia e a fisiologia dos órgãos sexuais } \\
\text { externos, em especial do feminino. O domínio de conteúdo } \\
\text { quanto às formas de transmissão e de sinais e sintomas } \\
\text { relacionados às infecções sexualmente transmissíveis foi } \\
\text { também reduzido. }\end{array}$ \\
\hline $\begin{array}{l}\text { 20.Maciel et al., } 2017 \text {, } \\
\text { Caracterização do comportamento } \\
\text { sexual entre adolescentes }\end{array}$ & $\begin{array}{l}\text { Descrever o comportamento } \\
\text { sexual dos adolescentes das escolas estaduais } \\
\text { do município de Senhor do Bomfim, Bahia. }\end{array}$ & Estudo quantitativo descritivo & $\begin{array}{l}\text { a maioria dos adolescentes já teve a primeira relação sexual, } \\
\text { aproximadamente um terço não utilizou } \\
\text { método contraceptivo na primeira relação e parcela } \\
\text { significativa já esteve grávida ou parceira engravidou. Apenas } \\
\text { metade dos jovens referiu fazer uso de contraceptivo em todas } \\
\text { as relações e o mais utilizado foi o preservativo. }\end{array}$ \\
\hline $\begin{array}{l}\text { 21. Santana. 2017, Práticas } \\
\text { sexuais de jovens universitários e } \\
\text { a vulnerabilidade às Infecções } \\
\text { Sexualmente Transmissíveis }\end{array}$ & $\begin{array}{l}\text { Objetivo geral analisar } \\
\text { a vulnerabilidade dos jovens universitários às Infecções } \\
\text { Sexualmente Transmissíveis (IST }\end{array}$ & $\begin{array}{l}\text { Tipo descritivo em abordagem } \\
\text { qualitativa, }\end{array}$ & $\begin{array}{l}\text { As condutas sexuais dos jovens têm sido mais liberais, e } \\
\text { baseadas em um estilo de vida de oportunidades. Em } \\
\text { decorrência destes fatos, os universitários se reconhecem como } \\
\text { uma população vulnerável, assunção de comportamentos } \\
\text { de risco como a não adoção do preservativo de modo continuo }\end{array}$ \\
\hline
\end{tabular}


Research, Society and Development, v. 10, n. 4, e20910414024, 2021

(CC BY 4.0) | ISSN 2525-3409 | DOI: http://dx.doi.org/10.33448/rsd-v10i4.14024

\begin{tabular}{|c|c|c|c|}
\hline & & & $\begin{array}{l}\text { em todos os intercursos sexuais, o uso de álcool e drogas que } \\
\text { favorecem a exposição do grupo às IST. }\end{array}$ \\
\hline $\begin{array}{l}\text { 22. Castro, Caldas, Morcillo, } \\
\text { Pereira e Velho, 2016, O } \\
\text { conhecimento e o ensino sobre } \\
\text { doenças sexualmente } \\
\text { transmissíveis entre universitários }\end{array}$ & $\begin{array}{l}\text { Buscou quantificar e gerar a } \\
\text { autopercepção do conhecimento (ou não) sobre as DST }\end{array}$ & Método quantitativo & $\begin{array}{l}\text { Entre os estudantes sexualmente ativos, } 26,9 \% \text { não tinham } \\
\text { parceiro fixo e } 28,2 \% \text { mais de dois parceiros por } \\
\text { ano. O preservativo era usado por } 99 \% \text { dos alunos, mas menos } \\
\text { de } 20 \% \text { o usava de forma adequada. }\end{array}$ \\
\hline $\begin{array}{l}23 \text { Rodrigues, 2016, Adolescentes } \\
\text { em situação de acolhimento } \\
\text { institucional: vulnerabilidade às } \\
\text { Infecções Sexualmente } \\
\text { Transmissíveis (IST) }\end{array}$ & $\begin{array}{l}\text { Analisar as dimensões } \\
\text { de vulnerabilidade às IST em adolescentes em situação } \\
\text { de acolhimento institucional, }\end{array}$ & $\begin{array}{l}\text { Pesquisa } \\
\text { qualitativa de caráter descritivo- } \\
\text { exploratório }\end{array}$ & $\begin{array}{l}\text { A pesquisa considerou que conhecer os roteiros sexuais e as } \\
\text { dimensões de vulnerabilidade de adolescentes em situação } \\
\text { de acolhimento às IST revelou que são questões fundamentais } \\
\text { a serem incorporadas na assistência a essas adolescentes, tendo } \\
\text { em vista a prevenção das IST e promoção da saúde sexual }\end{array}$ \\
\hline $\begin{array}{l}\text { 24. Costa, Araújo, Araújo, Gubert } \\
\text { e Vieira, 2015, Protagonismo de } \\
\text { adolescentes na prevenção de } \\
\text { doenças sexualmente } \\
\text { transmissíveis }\end{array}$ & $\begin{array}{l}\text { Analisar o protagonismo de adolescentes escolares } \\
\text { na prevenção de doenças sexualmente transmissíveis }\end{array}$ & $\begin{array}{l}\text { Estudo qualitativo do tipo pesquisa- } \\
\text { ação }\end{array}$ & $\begin{array}{l}\text { Os depoimentos revelaram que os adolescentes protagonizaram } \\
\text { suas participações no planejamento da intervenção educativa, } \\
\text { definindo e organizando o espaço educativo escolar, e } \\
\text { confeccionando materiais e temas a serem utilizados nas ações } \\
\text { de prevenção às doenças sexualmente transmissíveis, gerando } \\
\text { um sentimento de domínio sobre o assunto. }\end{array}$ \\
\hline $\begin{array}{l}\text { 25. Alves, } 2015 \text { Educação } \\
\text { permanente sobre infecção } \\
\text { sexualmente transmissível no } \\
\text { Instituto Federal Fluminense }\end{array}$ & $\begin{array}{l}\text { Identificar as dúvidas dos alunos do Instituto Federal } \\
\text { Fluminense (IFF) Campus Campus Guarus } \\
\text { sobre Infecção Sexualmente Transmissível (IST) e propor } \\
\text { uma abordagem ou metodologia educacional mais } \\
\text { apropriada para os mesmos; programar e realizar a Educação } \\
\text { Permanente, no Instituto Federal Fluminense, }\end{array}$ & $\begin{array}{l}\text { Estudo descritivo e exploratório, com } \\
\text { abordagem metodológica qualitativa, }\end{array}$ & $\begin{array}{l}\text { Desconhecimento + Infecção Sexualmente Transmissível } \\
\text { = Perigo; Educação e Saúde em Ambiente Escolar; Educação } \\
\text { Permanente na Escola; A importância da sensibilização para } \\
\text { reconstrução de atitudes e valores profissionais }\end{array}$ \\
\hline
\end{tabular}

Fonte: Autores. 


\section{Categoria 1: Início sexual precoce e adesão ao uso do preservativo}

Através das evidencias cientificas constatou-se que os comportamentos de risco mais presentes nos estudos da literatura foi a iniciação sexual precoce com o sexo masculino mais vulnerável, acompanhada de práticas sexuais desprotegida, sem adesão neste público ao uso frequente do preservativo, aumentando assim o risco das infecções sexualmente transmissíveis.

Os jovens iniciam sua vida sexual de forma cada vez mais precoce antes mesmo de adentrarem nas universidades, o que contribui para aquisição de IST, uma vez que em muitos casos são decisões imaturas, influenciadas por amigos, desprovidas de orientação. Sendo assim, é relevante que as atividades de prevenção sejam exploradas antecipadamente no ambiente familiar, nas escolas e espaços de convivências de adolescentes (Alves \& Aguiar, 2020).

A decisão em adiar ou antecipar o início da vida sexual nem sempre é fruto de vontade própria, muitas jovens baseiamse em normas sociais vigentes que preconizam a iniciativa sexual como prerrogativa masculina, cabendo à mulher resistir pelo maior tempo possível para posteriormente ceder (Costa et al., 2019; Brasil, Cardoso \& Silva, 2019).

Este estudo demonstrou uma concentração de comportamentos de risco entre os meninos. Sabe-se que socialmente há uma pressão social para comprovação da sua masculinidade, o que incentiva a iniciação precoce, o sexo casual e maior número de parceiros sexuais. Essa maior vulnerabilidade dos meninos deve ser levada em consideração ao se planejar intervenções para esse público específico, tendo em vista uma variação de adesão efetiva ao uso do preservativo encontrado nesse grupo (Costa et al., 2019).

A multiplicidade de parceiros e o não uso do preservativo nas relações sexuais estão em uma escala proximal para aquisição de IST, em contrapartida o álcool, uso de drogas e o fumo estão em um nível mais distal em escala de proximidade, entretanto podem ser a porta de entrada para o comportamento de risco contribuindo para o abandono do preservativo, o elevado número de parceiros e possíveis aquisições de IST (Lima, 2019)

Soma-se a isso o fato de que esse grupo populacional tem apresentado menor adesão ao uso de preservativo. Estudo que estimou a carga global de doenças em jovens de 10 a 24 anos aponta o sexo desprotegido como uma importante causa do aumento do número de anos de vida perdidos por incapacidade (DALYs) entre esses indivíduos (Felisbino-Mendes, Paula, Machado, Oliveira-Campos \& Malta, 2018).

A não adesão ao preservativo pelos adolescentes tem sido relacionada ao baixo nível de informação relacionada a classes sociais mais baixas, a credibilidade depositada no método ou sua banalização, crença na invulnerabilidade às infecções, menores sensações prazerosas, situações de marginalização social, natureza contestadora, não concordância do parceiro, dentre outros, o que contribui para maiores incidências de IST na população em questão (Sousa et al., 2018)

Os homens carregam consigo com maior frequência o preservativo, porém concordam que o uso preservativo atrapalha durante a relação sexual, o que muitas vezes favorece a negligência do uso, sendo mais impulsivos e adotam comportamento de risco para não perder a relação sexual. Em contrapartida as mulheres possuem um comportamento mais vulnerável devido a submissão de gênero (Baldoino, Silva, Ribeiro \& Ribeiro, 2018)

Ressalta-se que se tem observado aumento gradativo da incidência dessas infecções entre indivíduos muito jovens, principalmente sífilis e HIV/AIDS O preservativo é o único método que proporciona dupla proteção, contra as IST, incluindo HIV/AIDS, e contra gravidez (Oliveira, Abud, Inagaki, Alves \& Matos, 2018)

Vivências acerca da prevenção para IST/HIV/Aids, aponta que os participantes afirmaram saber como prevenir-se de tais infecções em suas vivências, concordando que o não uso do preservativo é o maior meio para contraí- las. Mesmo assim, muitos afirmaram que o preservativo masculino inibe o prazer, o que restringe o seu uso a momentos específicos em que se encontrem em situações que considerem de risco (Silva, Cortes, Espirito Santo \& Cordeiro, 2017)

A significância que os jovens atribuem ao método preventivo, a confiança afetiva em suas parcerias e o desconhecimento sobre o assunto mostra que as vivências pessoais eram preocupantes, especialmente por manterem ações e atitudes que os 
colocavam pessoalmente na situação de vulnerabilidade pela pouca prevenção para IST/HIV/|Aids. Em risco parece estar sempre o outro, levando-os a negar para si mesmos as suas vivências de risco (Cordeiro, Santos, Sales, Morais \& Dutra, 2017).

\section{Categoria 2: Déficit de conhecimento sobre saúde sexaul e ist}

O desenvolvimento da sexualidade nem sempre é acompanhada de um amadurecimento afetivo e cognitivo, o que torna a adolescência uma etapa de extrema vulnerabilidade a riscos, os quais estão muito ligadas as características próprias do desenvolvimento psicoemocional dessa fase de vida e não se encontram preparados para assumir essa responsabilidade dos cuidados preventivos com a saúde sexual (Bordignon et al., 2017)

As situações de violência, desestrutura familiar, exposição aos riscos e falhas ou incongruência no uso de preservativos que associado à adolescência contribuem para o aparecimento das IST. Estas situações são importantes, pois definem algumas vulnerabilidades comuns neste período da vida (Almeida et al., 2017)

Observou-se, ainda, um conhecimento limitado dos adolescentes sobre a anatomia e a fisiologia dos órgãos sexuais externos, em especial do feminino, e que os jovens de classes sociais mais baixas apresentam maior incidência de práticas sexuais desprotegidas devido a falta de informação sobre as formas de prevenção da saúde sexual.

O protagonismo juvenil e as questões da sexualidade, incluindo os direitos sexuais e reprodutivos de adolescentes e jovens, têm sido foco de inúmeras pesquisas e de reformulação de políticas públicas mediante os crescentes índices de Infecções Sexualmente Transmissíveis (IST), de gravidez na adolescência, de aborto e de outros desdobramentos' que perpassam essa temática (Silva et al., 2016)

Na puberdade, ocorrem transformações físicas e sexuais, que alteram rapidamente a autoimagem dos adolescentes, fazendo com que os familiares fiquem confusos e ansiosos quando o adolescente começa a ter mudanças no corpo ou adquirirem interesses sexuais evidentes (Silva et al., 2015; Sasaki et al., 2014).

Os riscos que permeiam a prática da relação sexual sem proteção são muitas vezes deixados de lado pelos jovens, existem dificuldades sobre conhecimento, a forma como se usa o preservativo e, ainda, sua relevância para a prevenção de agravos na ausência de sua utilização e na prevenção das IST (Oliveira Campos et al., 2014)

Adolescentes que não recebem educação sexual tendem a assumir comportamentos de risco, tais como a iniciação sexual precoce e a multiplicidade de parceiros sexuais, o que demonstra a extrema importância da escola no direcionamento de comportamentos benéficos entre os jovens (Malta et al., 2011)

A dificuldade em abordar a sexualidade dos jovens não está presente somente na família. Estudos evidenciam que o receio de julgamentos e da falta de confidencialidade das informações por parte dos profissionais de saúde, bem como a pouca habilidade destes em atender as necessidades dos jovens resulta no distanciamento deste grupo dos centros de saúde (Souza, 2011)

A demora em procurar os serviços de saúde após o início da vida sexual pode representar um período de risco continuado, o qual interfere na saúde sexual e reprodutiva dos adolescentes (Maciel et al., 2017)

O recebimento de orientação sexual da escola mostrou-se como fator favorável ao adiamento da primeira relação sexual. Os estudos evidenciaram que adolescentes que receberam da escola as primeiras informações sobre gravidez e contracepção foram menos propensas a engravidarem (Santana, 2017; Castro et al., 2016)

\section{Conclusão}

De acordo com os resultados das evidencias cientificas é imprescindível que haja a construção de novas intervenções conjuntas que contemplem o efetivo protagonismo dos adolescentes e jovens escolares no seu papel de responsável pela 
prevenção da sua saúde sexual, sem as quais não será possível mudar essa realidade de comportamentos de riscos adotados no início da vida sexual na adolescência.

A saúde reprodutiva é um estado de completo bem-estar físico, mental e social, implica nos processos e funções do sistema reprodutivo em todas as fases da vida. Como parte da saúde reprodutiva, a saúde sexual visa à melhoria da qualidade de vida e das relações pessoais, ao desenvolvimento sexual saudável, seguro e satisfatório, além da assistência às IST, deficiências e outras práticas de risco relacionadas à sexualidade.

Neste sentido se faz necessário o diálogo sobre sexo seguro e as IST que podem ser adquiridas quando o preservativo é negligenciado, com o objetivo de desenvolver no jovem a possibilidade de tomar decisões com segurança e responsabilidade, reduzindo assim vulnerabilidades.

\section{Referências}

Almeida, R. A. A. S., Corrêa, R. D. G. C. F., Rolim, I. L. T. P., Hora, J. M. D., Linard, A. G., Coutinho, N. P. S., \& Oliveira, P. D. S. (2017). Conhecimento de adolescentes relacionados às doenças sexualmente transmissíveis e gravidez. Revista Brasileira de Enfermagem, 70(5), 1033-1039.

Alves, L. D. S., \& Aguiar, R. S. (2020). Saúde sexual e infecções sexualmente transmissíveis na adolescência: uma revisão integrativa. Nursing (Säo Paulo), 3683-3687.

Alves, L. M. D. S. (2015). Educação permanente sobre infecção sexualmente transmissível no Instituto Federal Fluminense.

Araújo, E. C. (2001). Adoção de práticas de sexo mais seguro de jovens do sexo masculino. São Paulo (Doctoral dissertation, tese de Doutorado em Enfermagem]. São Paulo (SP): Programa de Pós-Graduação em Enfermagem da Universidade Federal de São Paulo).

Baldoino, L. S., Silva, S. M. D. N., Ribeiro, A. M. N., \& Ribeiro, E. K. C. (2018). Educação em saúde para adolescentes no contexto escolar: um relato de experiência. Rev. enferm. UFPE on line, 1161-1167.

Bordignon, M. N. F. D., Liberali, R., \& Bordignon, J. C. P. (2017). Causas da não utilização de preservativos nas práticas sexuais de adolescentes: revisão integrativa. Rev. enferm. UFPE on line, 207-213.

Brasil, M. E., Cardoso, F. B., \& Silva, L. M. D. (2019). Conhecimento de escolares sobre infecções sexualmente transmissíveis e métodos contraceptivos. Revista de Enfermagem UFPE On line, 13, e242261.

Castro, E. L. D., Caldas, T. A. D., Morcillo, A. M., Pereira, E. M. D. A., \& Velho, P. E. N. F. (2016). O conhecimento e o ensino sobre doenças sexualmente transmissíveis entre universitários. Ciência \& Saúde Coletiva, 21, 1975-1984.

Castro, G.C., Abramovay, M., Silva, L.B. (2014). Juventudes e sexualidade. UNESCO Brasil.

Cordeiro, J. K. R., Santos, M. M. D., Sales, L. K. O., Morais, I. F. D., \& Dutra, G. R. S. D. F. (2017). Adolescentes escolares acerca das DST/AIDS: quando o conhecimento não acompanha as práticas seguras. Rev. Enferm. UFPE on line, 2888-2896.

Costa, A. C. P. D. J., Araújo, M. F. M. D., Araújo, T. M. D., Gubert, F. D. A., \& Vieira, N. F. C. (2015). Protagonismo de adolescentes na prevenção de doenças sexualmente transmissíveis. Acta Paulista de Enfermagem, 28(5), 482-487.

Costa, M. I.F., Viana, T. R.F., Pinheiro, P. N.C., Cardoso, M. V. L.M.L., Barbosa, L.P., \& Luna, I.T. (2019). Determinantes sociais de saúde e vulnerabilidades às infecções sexualmente transmissíveis em adolescentes. Revista Brasileira de Enfermagem, 72(6).

Farias, J. C. D., Nahas, M. V., Barros, M. V. G. D., Loch, M. R., Oliveira, E. S. A. D., De Bem, M. F. L., \& Lopes, A. D. S. (2009). Comportamentos de risco à saúde em adolescentes no Sul do Brasil: prevalência e fatores associados. Revista Panamericana de Salud Pública, $25,344-352$.

Felisbino-Mendes, M. S., Paula, T. F. D., Machado, Í. E., Oliveira-Campos, M., \& Malta, D. C. (2018). Análise dos indicadores de saúde sexual e reprodutiva de adolescentes brasileiros, 2009, 2012 e 2015. Revista Brasileira de Epidemiologia, 21, e180013.

Joint United Nations Program on HIV/AIDS. (2016). Global Aids update. Geneva: Unaids

Lima, G. D. S. F. (2019). Práticas de prevenção de infecções sexualmente transmissíveis de universitários do sexo masculino: estudo comparativo.

Maciel, K. M. D. N., Andrade, M. S., Cruz, L. Z., Fraga, C. D. D. S., Paixão, G. P. D. N., \& Souza, R. S. (2017). Caracterização do comportamento sexual entre adolescentes. Rev. Enferm. UERJ, Rio de Janeiro, 25(e23496), 1-7.

Malta, D. C., Silva, M. A. I., Mello, F. C. M. D., Monteiro, R. A., Porto, D. L., Sardinha, L. M. V., \& Freitas, P. C. D. (2011). Saúde sexual dos adolescentes segundo a Pesquisa Nacional de Saúde dos Escolares. Revista brasileira de epidemiologia, 14, 147-156.

Mendes, K. D. S., Silveira, R. C. D. C. P., \& Galvão, C. M. (2008). Revisão integrativa: método de pesquisa para a incorporação de evidências na saúde e na enfermagem. Texto \& contexto-enfermagem, 17(4), 758-764

Ministério da Saúde (Brasil). (2016). Secretaria de Vigilância em Saúde, Departamento de DST, Aids e Hepatites Virais. Boletim epidemiológico HIV-AIDS. $5(1): 3-58$. 
Research, Society and Development, v. 10, n. 4, e20910414024, 2021

(CC BY 4.0) | ISSN 2525-3409 | DOI: http://dx.doi.org/10.33448/rsd-v10i4.14024

Ministério da Saúde (Brasil). (2019). Boletim Epidemiológico de AIDS, HIV e DST Boletim Epidemiológico Especial Secretaria de Vigilância em Saúde Ministério da Saúde. Brasília - DF.

Moura, L. R., Torres, L. M., Cadete, M. M. M., \& de Freitas Cunha, C. (2018). Factors associated with health risk behaviors among Brazilian adolescents. Revista da Escola de Enfermagem da USP, 52, e03304-e03304.

Oliveira, P. S. D., Abud, A. C. F., Inagaki, A. D. D. M., Alves, J. A. B., \& Matos, K. F. (2018). Vulnerabilidade de adolescentes às doenças sexualmente transmissíveis na atenção primária. Rev. enferm. UFPE on line, 753-762.

Oliveira-Campos, M., Nunes, M. L., Madeira, F. D. C., Santos, M. G., Bregmann, S. R., Malta, D. C., \& Barreto, S. M. (2014). Comportamento sexual em adolescentes brasileiros, Pesquisa Nacional de Saúde do Escolar (PeNSE 2012). Revista Brasileira de Epidemiologia, 17, 116-130.

Rodrigues, R. F. (2016). Adolescentes em situação de acolhimento institucional: vulnerabilidade às Infecções Sexualmente Transmissíveis (IST).

Santana, R. S. C. (2017). Práticas sexuais de jovens universitários e a vulnerabilidade às Infecções Sexualmente Transmissíveis.

Sasaki, R. S. A., Souza, M. M. D., Leles, C. R., Malta, D. C., Sardinha, L. M. V., \& Freire, M. D. C. M. (2014). Comportamento sexual de adolescentes escolares da cidade de Goiânia, Goiás. Revista Brasileira de Epidemiologia, 17, 172-182.

SBP - Sociedade Brasileira de Pediatria Departamentos Científicos de Adolescência e Infectologia. (2018). Infecções Sexualmente Transmissíveis na adolescência, 6 .

Silva, A. T. D., Jacob, M. H. V. M., \& Hirdes, A. (2015). Conhecimento de adolescentes do ensino médio sobre DST/AIDS no sul do Brasil. Aletheia, (46), 3449.

Silva, L. M., Cortes, E. A., Espírito Santo, J. N., \& Cordeiro, B. C. (2017). Pesquisa-ação: promovendo educação em saúde com adolescentes sobre infecção sexualmente transmissível. Revista de Enfermagem UFPE on line, 11(9), 3642-3649.

Silva, R. A. R. D., Nelson, A. R. C., Duarte, F. H. D. S., Prado, N. C. D. C., Holanda, J. R. R., \& Costa, D. A. R. D. S. (2016). Conhecimento de estudantes adolescentes sobre transmissão, prevenção e comportamentos de risco em relação as DST/HIV/AIDS.

Soares, S. M., Amaral, M. A., Silva, L. B., \& Silva, P. A. B. (2008). Workshops on sexuality in adolescence: revealing voices, unveiling views student's of the medium teaching glances. Esc Anna Nery, 12(3), 485-91.

Sousa, B. C. D., Santos, R. S. D., Santana, K. C., Souzas, R., Leite, Á. J. M., \& Medeiros, D. S. D. (2018). Comportamento sexual e fatores associados em adolescentes da zona rural. Revista de Saúde Pública, 52, 39.

Souza, V. D. (2011). Adolescentes em cena: uma proposta educativa no campo da saúde sexual e reprodutiva. Revista da Escola de Enfermagem da USP, 45(2), 1716-1721. 